\title{
PROBLEMA DE ROTEIRIZAÇÃO DE VEÍCULOS COM MULTI- COMPARTIMENTOS COM COLETA E ENTREGA MISTA RESTRITA
}

\author{
Carlos Leonardo Ramos Póvoa \\ Universidade Estadual do Norte Fluminense (UENF) \\ Av. Alberto Lamego, 2000 - Parque Califórnia - Campos dos Goytacazes - RJ \\ clrp@uenf.br \\ Geraldo Galdino de Paula Jr. \\ Universidade Estadual do Norte Fluminense (UENF) \\ galdino@uenf.br \\ Laura Moreira Vasconcelos Lima da Silva \\ Universidade Estadual do Norte Fluminense (UENF) \\ lauramoreira@pq.uenf.br \\ Júlia Landeira Moreira \\ Universidade Estadual do Norte Fluminense (UENF) \\ julialandeira98@gmail.com
}

\begin{abstract}
RESUMO
Este artigo apresenta o problema de roteirização de veículos com multi-compartimentos com coleta e entrega mista restrita (MC-PRVCEMR). O mesmo é uma generalização do problema de roteirização de veículos com entregas e coletas, possibilitando o decisor a escolher o quanto de capacidade livre do veículo será aceito antes de começar a fazer as coletas. Uma heurística GRASP foi desenvolvida para solução do problema, bem como um procedimento de verificação de inserção do cliente na rota. $\mathrm{O}$ algoritmo foi testado com 46 instâncias, e apesar da utilização de um procedimento simples de busca local, o mesmo apresentou resultados compatíveis com a literatura.
\end{abstract}

Palavra-chave: Logística; Transporte; Roteirização de Veículos; Heurística; Otimização

\begin{abstract}
This paper presents the problem of vehicle routing with multi-compartments with pickup and restricted mixed delivery (MC-PRVCEMR). The same is a generalization of the problem of vehicle routing with deliveries and pickups, allowing the decision maker to choose how much free capacity of the vehicle will be accepted before starting to make the pickups. A GRASP heuristic has been developed to solve the problem, as well as a procedure to verify the customer's insertion in the route. The algorithm was tested with 46 instances, and despite the use of a simple local search procedure, it presented results compatible with the literature.
\end{abstract}

Keywords: Logistics; Transportation; Routing; Heuristic; Optimization 


\section{SPOLM2O19}

XIX SIMPÓSIO DE PESQUISA OPERACIONAL ELOGÍSTICA DA MARINHA RIO DE JANEIRO, RJ, BRASIL - 06 A 08 DE NOVEMBRO DE 2019

\section{INTRODUÇÃO}

O problema clássico de roteirização de veículos (PRV) consiste em definir roteiros que minimizem o custo total de atendimento, cada um dos quais iniciando e terminando no depósito ou base de veículos, assegurando que cada cliente seja visitado, ou seja, tenha suas mercadorias entregues, exatamente uma vez e a demanda em qualquer rota não exceda as restrições do veículo que a atenda (LAPORTE ET AL., 2000).

O problema de roteirização de veículos com coleta e entrega difere do problema clássico de roteirização por permitir que os veículos façam entregas e coletas de mercadorias. O problema aparece pela primeira vez no trabalho de Deif e Bodin (1984) e pode ser enquadrado como um problema de otimização aplicado a logística reversa, que visa planejar o transporte de produtos aos clientes, bem como o retorno de mercadorias ou embalagens (resíduos) para centros de reciclagem ou depósitos. Um exemplo prático são as indústrias de alimentos, na qual as embalagens devem ser coletadas para reciclagem. Outro exemplo pode ser encontrado na distribuição de bebidas, dentre outros.

Nesse problema de roteirização, o conjunto de clientes a serem atendidos são particionados em dois, os que requerem entrega (linehaul), e os que demandam coleta (backhaul). O problema pode ser dividido em quatro classes, segundo o trabalho de Parragh et al. (2008). Na primeira classe, os clientes de entrega devem ser visitados antes dos clientes de coleta, esse problema é conhecido na literatura como vehicle routing with backhauls (PRVB). Na segunda, os clientes de coleta são permitidos em qualquer ponto da rota, desde que a capacidade do veículo não seja violada, problema esse conhecido como roteirização de veículos com coleta e entrega mista (PRVCEM). Na terceira classe, clientes que demandam coleta e entrega podem ser visitados duas vezes. Na quarta, tem-se o caso de coleta e entrega simultânea, onde os consumidores demandam coleta e entrega ao mesmo tempo. Essa questão é conhecida na literatura como problema de roteirização de veículos com coleta e entrega simultânea (PRVCES).

O problema de roteirização com coleta e entrega mista foi estudado pela primeira vez por Wade e Salhi (2002). Os autores apresentam uma heurística de inserção gulosa para resolver o problema. Experimentos computacionais foram feitos com instâncias PRVB e foi demostrado que melhorias podem ser alcançadas pela incorporação da mistura entre as coletas e entregas em qualquer momento da rota.

Crispim e Brandão (2005) propuserem um algoritmo híbrido para a solução do PRVCEM e conseguiram melhores soluções em todas as instâncias de Salhi e Nagy (1999). Ropke e Pisinger (2006) usaram ALNS (Adaptive Large Neighborhood Search) para resolver o problema e obtiveram uma redução de $10 \%$ nas mesmas instâncias.

Estudos mais atuais visam correlacionar o problema de roteirização de veículos com coleta e entrega a questões ambientais. Turkensteen e Hasle (2017) realizam uma avaliação dos efeitos da emissão de carbono na atmosfera, constatando que entregas e coletas mistas geram economias de $20 \%$ a $40 \%$ dessa ameaça ao meio ambiente.

O objetivo do presente trabalho é descrever o problema de roteirização de veículos com multi-compartimentos com coleta e entrega mista restrita, bem como o desenvolvimento de uma heurística GRASP para a solução do mesmo. Experimentos computacionais foram realizados para avaliar o algoritmo. Destaca-se que não existe referência a esse problema na literatura até o momento. 
O artigo é estruturado em quatro seções, além da introdução. A segunda seção é dedicada em descrever o problema de roteirização de veículos com multi-compartimentos com coleta e entrega mista restrita; a terceira apresenta o algoritmo GRASP desenvolvido para resolver o problema; a quarta seção apresenta os experimentos computacionais com instâncias da literatura; e por fim, na última seção são feitas as conclusões.

\section{DEFINIÇÃO DO PROBLEMA}

O presente estudo foca na classe do problema de roteirização com coleta, conhecida como mista e restrita. Alguns pesquisadores optam por seguir a linha que aponta que um veículo não pode realizar coletas até todas as entregas serem feitas. De acordo com Nagy et al. (2013), no Problema de Roteirização de Veículos com Coleta e Entrega Mista Restrita, supõe-se que o veículo sai do depósito carregando somente os itens a serem entregues, passando a realizar coletas em algum momento da rota, fazendo com que ocorra uma mistura das mercadorias, finalizando com o veículo chegando novamente ao depósito somente com as coletas realizadas. Para isso, faz-se necessário checar a capacidade do veículo a todo momento, já que o nível de mercadorias pode aumentar ou diminuir de acordo com a ordem dos clientes de entrega e coleta na rota, explicitando uma questão mais complexa a ser resolvida.

O problema proposto está sujeito a uma restrição de capacidade do veículo, como mencionado anteriormente. Casco et. al (1988) apresenta a existência do fator $\gamma$, que indica a capacidade livre do veículo disponível antes das coletas. Pode-se notar que quando o $\gamma$ é $100 \%$, o veículo precisa estar totalmente livre para começar a realizar as coletas, isto é, o problema é reduzido para o Problema de Roteirização de Veículos com Backhauls (PRVB), o qual as entregas são feitas por completo antes do início das coletas, perdendo o caráter misto do estudo. Já com $\gamma$ igual a $0 \%$, o caminhão tem disponibilidade de realizar entregas e coletas a todo momento, respeitando apenas a sua capacidade máxima, caracterizando o Problema de Roteirização de Veículos com Coleta e Entrega Mista (PRVCEM). Quando o $\gamma$ se encontra entre $0 \%$ e $100 \%$, o problema é denominado Problema de Roteirização de Veículos com Coleta e Entrega Mista Restrita (PRVCEMR), demandando uma determinada capacidade livre do veículo para iniciar as coletas.

Considera-se três casos ilustrativos dos problemas descritos acima, sendo o primeiro uma representação do Problema de Roteirização de Veículos com Backhauls (PRVB), com o $\gamma$ igual a 100\%; o segundo indicando o Problema de Roteirização de Veículos com Coleta e Entrega Mista (PRVCEM), com o $\gamma$ igual a $0 \%$, que permite entregas e coletas totalmente alternadas, desde que não exceda a capacidade total do veículo; e por fim, o último caso caracteriza o Problema de Roteirização de Veículos com Coleta e Entrega Mista Restrita (PRVCEMR), com o $\gamma$ escolhido de 50\%, fazendo com que o veículo precise da metade de sua capacidade livre para iniciar as coletas.

Nos exemplos das Figuras 1, 2 e 3, o depósito, identificado pela cor verde, está situado no ponto $(0,1)$. Há três clientes de entrega localizados nos pontos $(1,1),(1,0)$ e $(2,0)$, preenchidos de azul, com demanda única. Os clientes de coleta estão representados pela cor laranja e se encontram nos pontos $(0,0)$ e $(2,1)$, cada um possuindo uma unidade a ser coletada. $\mathrm{O}$ veículo possui um único compartimento e a capacidade máxima é de 3 unidades. A distância entre os nós é unitária. 
Na Figura 1, a rota 0-1-3-2-4-5-0 possui distância de $5+\sqrt{5} \simeq 7,24$, já que independente da posição dos nós de entrega e coleta, por ser PRVB $(\gamma=100 \%)$, é necessário esvaziar totalmente o veículo para iniciar as coletas. $\mathrm{Na}$ Figura 2, a partir do $\operatorname{PRVCEM}(\gamma=0 \%)$, a rota $0-1-4-2-3-5-0$ possui tamanho de 6 , observando uma melhoria de aproximadamente $17 \%$ se comparado com o PRVB. Na última exemplificação, a Figura 3 mostra que a distância da rota $0-1-2-4-3-5-0$ é de $4+2 \sqrt{2} \simeq 6,83$, com melhoria de aproximadamente $12 \%$ se comparada ao PRVB, já que agora se trata do PRVCEMR $(\gamma=$ $50 \%)$.

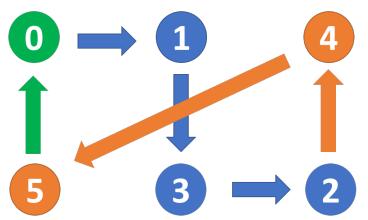

Figura 1 - PRVB $(\gamma=100 \%)$

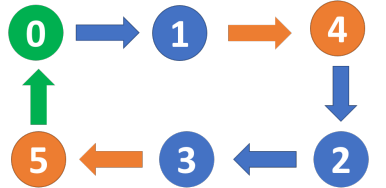

Figura 2 - PRVCEM $(\gamma=0 \%)$

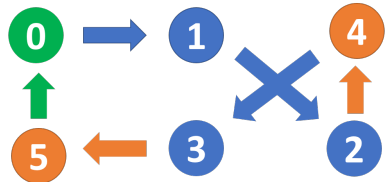

Figura 3 - PRVCEMR $(\gamma=50 \%)$

O problema de roteirização de veículos com multi-compartimentos com coleta e entrega mista restrita (MC-PRVCEMR) pode ser definido da seguinte forma: dado um grafo não direcionado $G=(P, E)$, o conjunto de vértices $P=\{0,1, \ldots, n\}$ representa os pontos nos quais os veículos deverão visitar e é composto por clientes de entrega $(L)$ e clientes que demandam coleta $(B)$, sendo o elemento $(\{0\})$ a base de operações onde os veículos irão partir. Os clientes serão visitados por um conjunto de veículos $V=\{1,2, \ldots, v\}$ não idênticos com diferentes custos e com diferentes capacidades $Q$. Cada veículo possui seu conjunto de compartimentos $C=\{1, \ldots, m\}$. O conjunto de arestas $E=\{(i, j) \in P, i<j\}$ representa as ligações entre os vértices e tem um custo de deslocamento não negativos $c_{i j}=$ $c_{j i}$. Todos esses custos satisfazem a desigualdade triangular. $\mathrm{O}$ objetivo é definir os roteiros de entrega que minimizem o custo total de atendimento, respeitando as restrições dos veículos (capacidade total e a cubagem de cada compartimento), assegurando que todo cliente seja visitado.

Para visualizar o problema descrito acima, outro exemplo é dado, no qual o depósito também é identificado pela cor verde, os três clientes de entrega estão pintados de azul e os de coleta estão representados pela cor laranja. $\mathrm{O}$ veículo, nesse caso, possui dois compartimentos, cada um com com capacidade de 2 unidades, totalizando a capacidade máxima total de 4 . O cliente 1 utiliza o compartimento $A$ e os clientes 2 e 3 , o compartimento B, ambos com demanda de uma unidade de entrega. Em se tratando de coletas (backhauls), percebe-se que o cliente 4 coleta 2 unidades, sendo uma no compartimento A e outra no compartimento B. O cliente 5 possui demanda de coleta de uma unidade no compartimento B. A distância entre os nós é unitária. A partir da Figura 4, é interessante observar que para $\gamma=0 \%$ no caso de multi-compartimento, a rota $0-1-4-2-3-5-0$ se torna inviável devido à demanda do cliente 4 de uma unidade coletada em cada compartimento. Com isso, uma rota alternativa foi encontrada, de tamanho $4+2 \sqrt{2} \simeq 6,83$, demonstrada pela sequência 0-3-2-4-1-5-0.

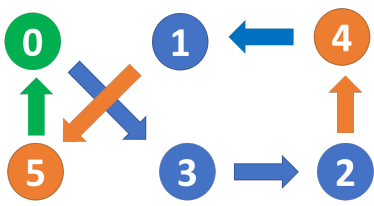

Figura 4 - MC-PRVCEMR $(\gamma=0 \%)$ 
O problema de roteirização de veículos com multi-compartimentos é caracterizado como NP-hard, uma vez que é considerado uma generalização do problema clássico de roteirização. Apenas instâncias pequenas podem ser solucionadas de forma exata. Essa é a justificativa do uso de heurísticas para a solução de problemas reais (EL FALLAHI et al., 2008). Na próxima seção é apresentado o algoritmo proposto para a solução do problema.

\section{ALGORITMO GRASP}

O algoritmo GRASP (Greedy Randomized Adaptive Search Procedures) é um método iterativo, proposto por Feo e Resende (1995), que consiste de duas fases: uma fase de construção, na qual uma solução viável é gerada a cada iteração, elemento a elemento, e uma fase de busca local na qual a solução é melhorada. O resultado final é a melhor solução obtida nas diferentes iterações do procedimento.

O algoritmo desenvolvido (Algoritmo 1) é baseado no trabalho de Chaovalitwongse et al. (2003) e Kontovarids e Bard (1995). A construção de soluções viáveis é iniciada pelo procedimento seleção de sementes (Algoritmo 2), que tem como objetivo inicializar cada nova rota com um consumidor. Esta seleção é feita de modo que os consumidores mais dispersos sejam alocados primeiro e o número de rotas iniciais seja igual ao número mínimo de veículos $(I)$ necessários para que todos os clientes sejam atendidos.
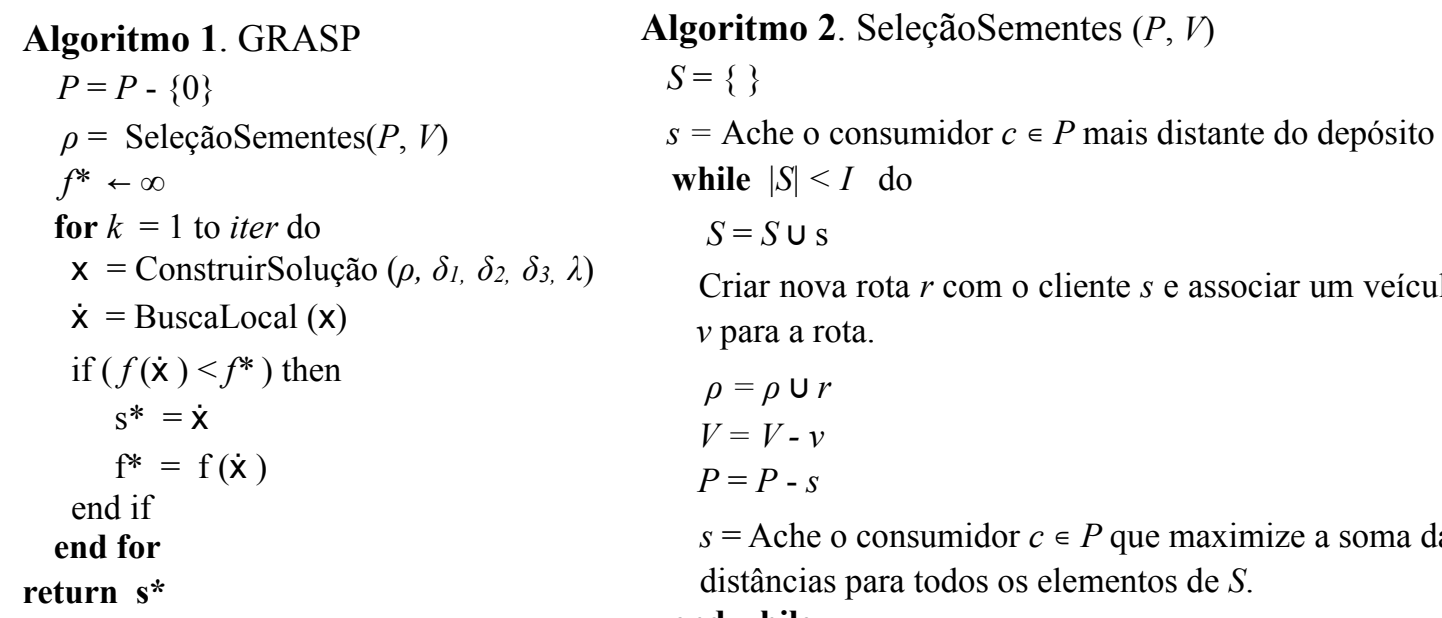

Criar nova rota $r$ com o cliente $s$ e associar um veículo viável $v$ para a rota.

$\rho=\rho \cup r$

$V=V-v$

$P=P-S$

$s=$ Ache o consumidor $c \in P$ que maximize a soma das distâncias para todos os elementos de $S$. end while

return $\rho$

Após o procedimento de seleção de sementes, o algoritmo de construção solução (Algoritmo 3) é chamado. O custo de inserção do consumidor $k$, na rota $r$, utilizando o veículo $v$, entre os consumidores $i$ e $j$ é designado por $C_{i, j, k, r, v}=\delta_{1} c_{i, j, k, r, v}^{1}+\delta_{2} c_{i, j, k, r, v}^{2}+\delta_{3} c_{i, j, k, r, v}^{3}$ As variáveis $\delta_{1}, \delta_{2}, \delta_{3}$ são pesos não negativos que irão ponderar os valores de cada componente da função custo e $\delta_{1}+\delta_{2}+\delta_{3}=1$. A primeira componente $c_{i, j, k, r, v}^{1}=V F E_{v}-\sum_{c=1}^{m} q_{k}^{c}$, sendo $V F E_{v}$ a capacidade livre do veículo $v$ e $q_{k}^{c}$ é a demanda de cada compartimento $c$ do cliente $k$, se o cliente for de coleta: $q_{k}^{c}=-q_{k}^{c}$. Essa função designa o custo associado à capacidade livre do veículo e mapeia grandes demandas em pequenos custos, consumidores com altas demandas devem ser associados aos veículos primeiro. Essa lógica é a mesma utilizada para problemas da mochila (Martello e Toth, 1990). A segunda componente é dada por $c_{i, j, k, r, v}^{2}=\left(d_{i k}+d_{j k}-d_{i j}\right) \alpha_{v}$, sendo $\alpha_{v}$ o custo por distância do veículo $v$. Esta componente 
mede o custo do aumento da distância com a inserção do consumidor $k$. A terceira componente insere o custo fixo do veículo $v$ e é dada por $c_{i, j, k, r, v}^{3}=F_{v}$.

O algoritmo 3 apresenta a fase de construção da GRASP e sua execução considera inicialmente achar o menor custo de inserção viável em cada rota $r \in \rho$, para cada consumidor $c \in P$ não associado, utilizando o veículo $v \in V$. Então é calculado uma penalidade $P_{c}$, que mede o custo que se deve pagar posteriormente, se o consumidor não for associado a sua rota de menor custo. Consumidores com altos valores de penalização devem ser associados a uma rota primeiro, enquanto os de menores valores podem esperar. A lista de candidatos restritos (LCR) é construída com os $\lambda$ maiores custos de penalização e o cliente a ser roteirizado é escolhido de forma aleatória.

O procedimento de busca local consiste em realocar os consumidores para uma rota de menor custo. $\mathrm{O}$ procedimento é inicializado com as rotas que possuem menos clientes. Se alguma rota apresentar no final uma quantidade nula de clientes, a mesma é eliminada da solução, liberando a utilização do veículo. Todas as restrições devem ser verificadas antes da mudança de rota. Se algum veículo for incompatível, tenta-se trocar os veículos das rotas ou utilizar algum outro veículo disponível. A seção 3.1 mostra o procedimento utilizado para verificar a viabilidade de inserção do cliente em uma rota específica.

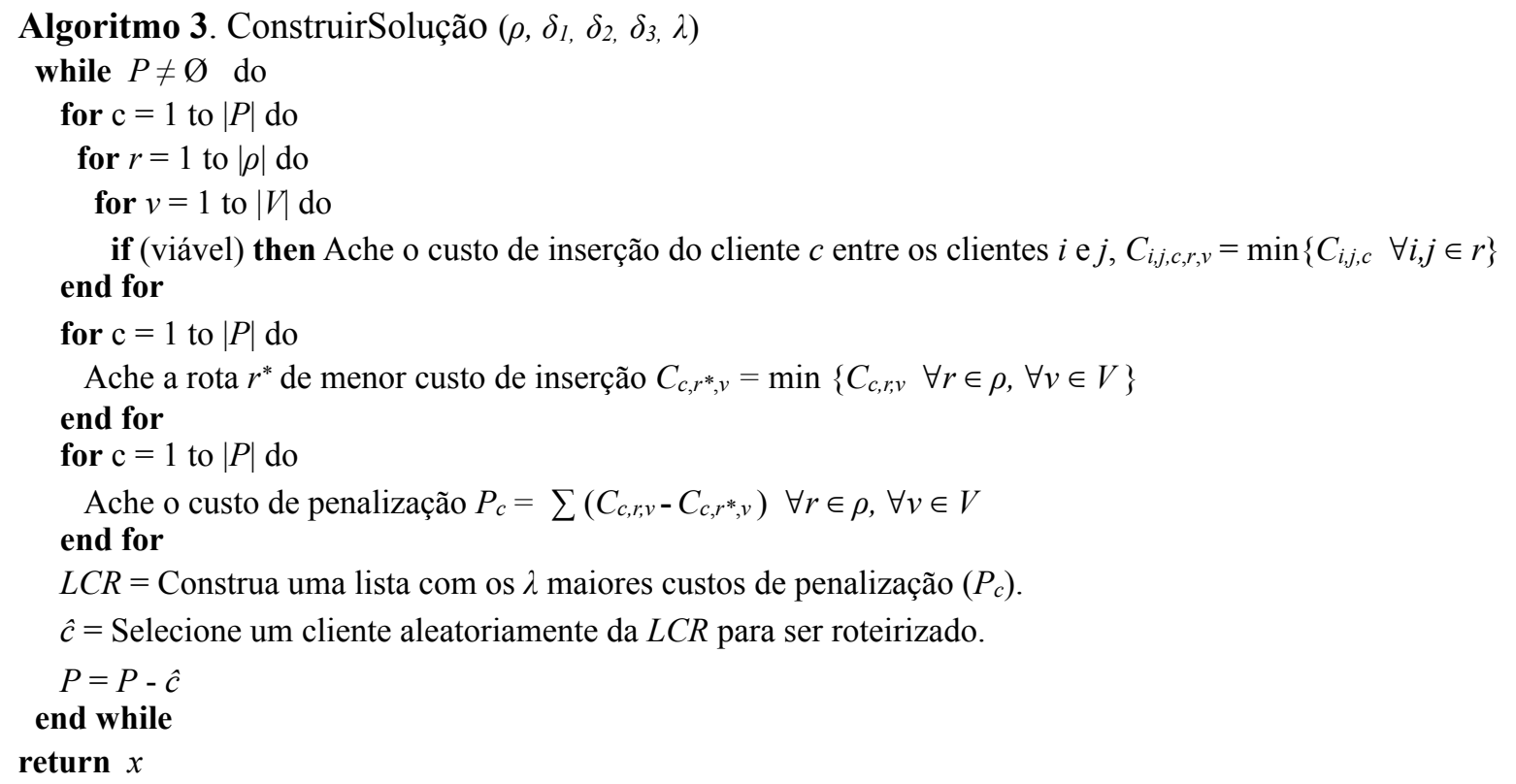

\subsection{CONDIÇÃo DE VIABILIDADE}

Checar a viabilidade de inserção do cliente em uma certa posição da rota é mais difícil no caso de coleta e entrega mista restrita que no problema clássico de roteirização (PRV). No Problema de Roteirização de Veículos com Backhauls (PRVB), o qual as entregas são feitas por completo antes do início das coletas, basta checar se a demanda total de entregas não ultrapassa a capacidade do veículo, idem para as coletas. No caso do PRVCEMR é mais complexo, pois a demanda de coleta e a capacidade livre do veículo varia de arco para arco. No caso do MC-PRVCEMR além de checar a capacidade total do veículos, temos que checar a capacidade de cada compartimento. 
Baseado no trabalho de Nagy et al. (2013) foi desenvolvido o seguinte procedimento para verificar a viabilidade de inserção do cliente $i$, na rota $r$ :

$T D=$ Demanda de entregas na rota $r ; T P=$ Demanda de coletas na rota $r$;

$Q_{v}=$ Capacidade total de carga do veículo $v$;

$Q_{v}^{c}=$ Capacidade total do compartimento $c$ do veículo $v$;

$q_{i}=$ Demanda total do cliente $i$;

$n=$ Total de clientes na rota $r$

$R_{l}=$ Restante a ser entregue a partir do cliente $l$;

$P_{k}=$ Total de coleta até o cliente $k$;

\section{a) Se o cliente $\boldsymbol{i}$ for de Entrega:}

\section{Se $\left(T D+q_{i} \leq Q_{v}\right) \mathbf{E}\left(T D^{c} \leq Q_{v}^{c} \quad \forall c \in C\right)$ então}

Se a Rota só conter clientes de entrega, o cliente $i$ pode ser inserido em qualquer posição.

Se a rota conter clientes de entrega e coleta então

Verificar em cada arco $k l$ a partir do primeiro cliente de coleta até o fim da rota (forward):

Se $\left(P_{k}+R_{l}+q_{i} \leq(1-\gamma) Q_{v}\right) \mathbf{E}\left(P_{k}^{c}+R_{l}^{c}+q_{i}^{c} \leq Q_{v}^{c} \quad \forall c \in C\right)$

$\mathrm{O}$ cliente $i$ pode ser inserido no arco $\mathrm{kl}$

\section{Se não}

Parar a busca, o cliente não pode ser inserido em nenhum outro arco subsequente

\section{fim se}

\section{fim se}

* O cliente de entrega $i$ pode ser inserido em qualquer arco antes do primeiro cliente de coleta.

\section{fim se}

\section{b) Se o cliente $\boldsymbol{i}$ for de Coleta:}

Se $\left(T P+q_{i} \leq Q_{v}\right) \mathbf{E}\left(T P^{C} \leq Q_{v}^{c} \forall c \in C\right)$ então

Se a Rota só conter clientes de coleta, o cliente $i$ pode ser inserido em qualquer posição.

Se a rota conter clientes de entrega e coleta então

Verificar em cada arco $\mathrm{kl}$ a partir do último cliente de entrega até o início da rota (backward):

Se $\left(P_{k}+R_{l}+q_{i} \leq(1-\gamma) Q_{v}\right) \mathbf{E}\left(P_{k}^{c}+R_{l}^{c}+q_{i}^{c} \leq Q_{v}^{c} \quad \forall c \in C\right)$

$\mathrm{O}$ cliente $i$ pode ser inserido no arco $\mathrm{kl}$

\section{Se não}

Parar a busca, o cliente não pode ser inserido em nenhum outro arco subsequente

fim se

\section{fim se}

* O cliente de coleta $i$ pode ser inserido em qualquer arco depois do último cliente de entrega.

\section{fim se}




\section{EXPERIMENTOS COMPUTACIONAIS}

Foi utilizado o conjunto de problemas proposto por Goetschalckx e Jacobs-Blecha (1989) elaborado para o PRVB, contendo 46 instâncias, com o número de clientes variando entre 25 e 150 e com um único compartimento de carga. A proporção de clientes com coleta é de $1 / 5$ (A, D, G, J, M), 1/3 (B, E, H, K, N) e 1/2 (C, F, I, L).

As instâncias foram resolvidas utilizando os seguintes parâmetros: $\lambda=5 ;$ iter $=300 ; \delta_{1}=0.1 ; \delta_{2}=0.8 ; \delta_{3}=0.1$; Os valores de $\gamma: 0,10,25,50,75$ e $100 \%$ foram utilizados nos testes. O procedimento GRASP foi implementado utilizando técnicas de orientação a objetos na linguagem object pascal e os experimentos foram processados em um PC Intel Core i5-2400 at 3.1 Ghz.

Os melhores resultados alcançados para os problemas PRVB $(\gamma=100 \%)$ e PRVM $(\gamma=0 \%)$ foram obtidos dos trabalhos de Brandão (2006), Halse (1992), Mingozzi et al. (1999), Nagy and Salhi (2005), Ropke and Pisinger (2006), Toth and Vigo (1997), Tütüncü et al. (2009), Wade and Salhi (2003), Wassan (2007), Wassan et al. (2008), Gajpal and Abad (2009) e Nagy et al. (2013).

Os resultados utilizando os outros valores de $\gamma$ foram comparados com o trabalho de Nagy et al. (2013). A Tabela 1 apresenta os resultados. Mesmo utilizando um procedimento de busca local muito simples, o algoritmo desenvolvido obteve melhores resultados em 6 instâncias em comparação com os melhores valores da literatura para o problema de PRVB $(\gamma=100 \%)$, já para o caso do PRVM $(\gamma=0 \%)$ obteve 9 melhores resultados. Para os outros valores de $\gamma(75 \%, 50 \%, 25 \%$ e $10 \%)$ foram obtidos $29,15,11$ e 12 melhores resultados respectivamente.

Tabela 1 - Resultados

\begin{tabular}{|c|c|c|c|c|c|c|c|c|c|c|c|c|c|c|c|}
\hline \multirow[t]{2}{*}{ Instância } & \multicolumn{4}{|l|}{$n$} & \multicolumn{2}{|c|}{$\gamma=75 \%$} & \multicolumn{2}{|c|}{$\gamma=50 \%$} & \multicolumn{2}{|c|}{$\gamma=25 \%$} & \multicolumn{2}{|c|}{$\gamma=10 \%$} & \multicolumn{3}{|c|}{$\gamma=0 \%$} \\
\hline & & Autor & $\begin{array}{c}\text { Melhor } \\
\text { PRVB }\end{array}$ & GRASP & Nagy & GRASP & Nagy & GRASP & Nagy & GRASP & Nagy & GRASP & Autor & $\begin{array}{l}\text { Melhor } \\
\text { MIXED }\end{array}$ & GRASP \\
\hline A1 & 25 & GR & 229886 & 229399 & 229884 & 229494 & 228023 & 228514 & 225341 & 226711 & 224307 & 225190 & GR & 223085 & 222442 \\
\hline A2 & 25 & GR & 180119 & 179255 & 180450 & 178959 & 172511 & 174982 & 169970 & 171235 & 169500 & 168273 & GR & 169497 & 165519 \\
\hline A3/A4 & 25 & $\mathrm{TV}$ & 155796 & 158739 & 154523 & 158739 & 149516 & 150827 & 143283 & 144761 & 142034 & 142034 & GR & 142032 & 142032 \\
\hline B1 & 30 & GR & 239080 & 222171 & 241197 & 229315 & 238988 & 226994 & 234027 & 231226 & 232436 & 224209 & GR & 232430 & 227700 \\
\hline B2 & 30 & GR & 198048 & 193070 & 202715 & 192604 & 193196 & 194990 & 182231 & 184585 & 182231 & 175997 & GR & 179194 & 175080 \\
\hline B3 & 30 & TV & 169372 & 175561 & 169190 & 173542 & 153774 & 160515 & 146709 & 151336 & 145702 & 145702 & E & 145699 & 145702 \\
\hline $\mathrm{C} 1$ & 40 & TV & 249448 & 252878 & 250557 & 252700 & 246189 & 252386 & 244184 & 250996 & 240246 & 251711 & WN & 237100 & 250954 \\
\hline $\mathrm{C2}$ & 40 & TV & 215020 & 220646 & 224794 & 222640 & 211991 & 217205 & 209353 & 209619 & 202183 & 206550 & WS & 196883 & 206448 \\
\hline $\mathrm{C} 3 / \mathrm{C} 4$ & 40 & TV & 195367 & 202835 & 207024 & 199925 & 190696 & 184546 & 174480 & 176731 & 176263 & 172891 & WN & 164794 & 171469 \\
\hline D1/D2 & 38 & $\mathrm{TV}$ & 316709 & 329228 & 314618 & 332627 & 314618 & 335121 & 311266 & 331155 & 308696 & 327664 & WN & 307109 & 323920 \\
\hline D3 & 38 & $\mathrm{TV}$ & 239479 & 250862 & 236664 & 246390 & 233652 & 250752 & 226232 & 243073 & 225336 & 233546 & WN & 220700 & 228785 \\
\hline D4 & 38 & TV & 205832 & 207736 & 210480 & 209543 & 195368 & 198370 & 188411 & 185911 & 182928 & 184515 & GR & 182496 & 180401 \\
\hline E1 & 45 & TV & 238880 & 257014 & 238747 & 257958 & 238747 & 253657 & 231073 & 242157 & 224650 & 239074 & WN & 220742 & 239886 \\
\hline E2 & 45 & TV & 212263 & 213995 & 223974 & 213058 & 207579 & 199368 & 197050 & 191275 & 198476 & 190049 & GR & 190048 & 190048 \\
\hline E3 & 45 & GR & 206659 & 205428 & 229597 & 206818 & 192305 & 192862 & 188968 & 186165 & 186525 & 185308 & WS & 182804 & 182987 \\
\hline
\end{tabular}


Tabela 1 - Continuação

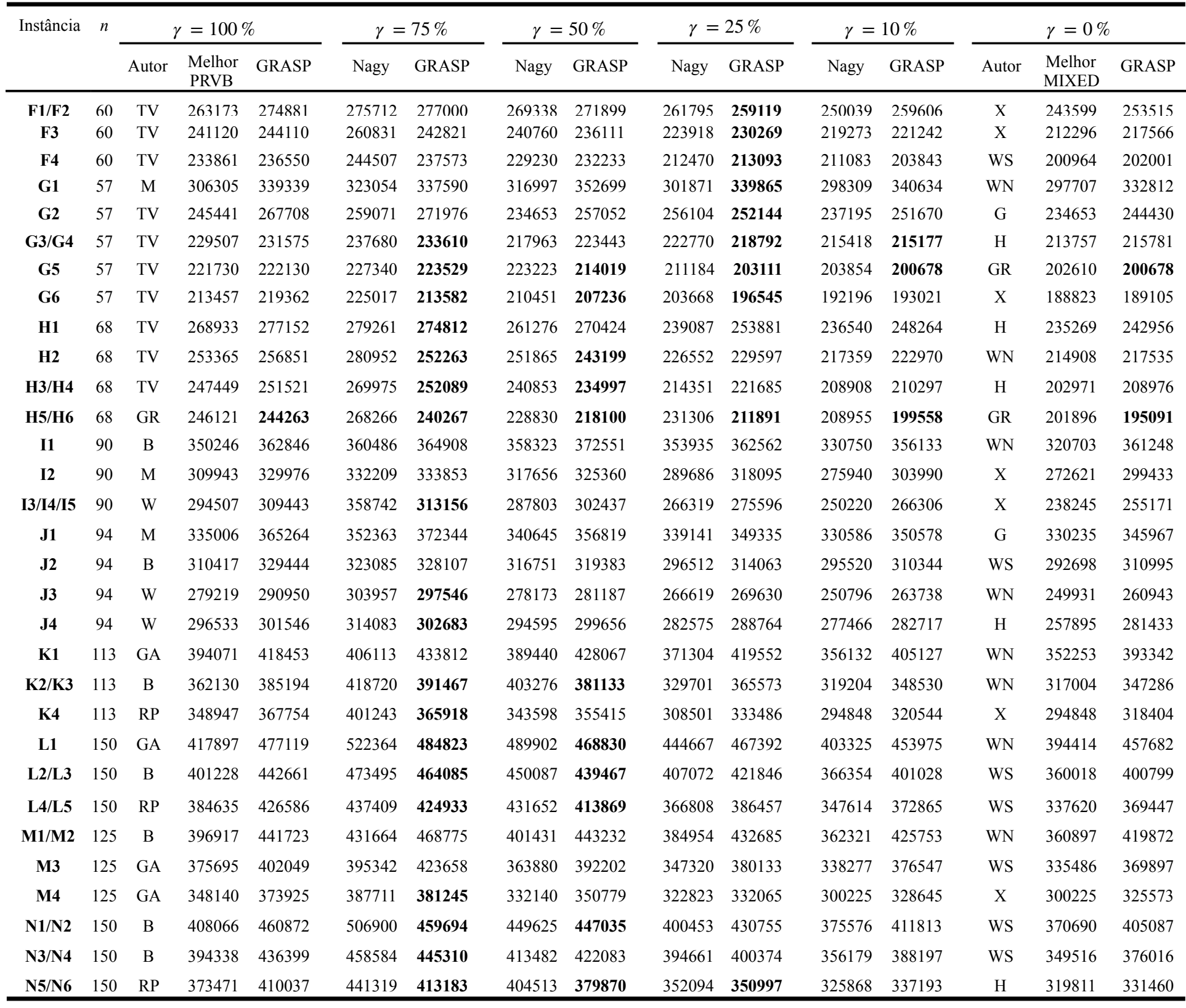

$n$ total de clientes; GR heurística GRASP elaborada nesse artigo; B Brandão (2006); GA Gajpal and Abad (2009); H Halse (1992); M Mingozzi et al. (1999); RP Ropke and Pisinger (2006); TV Toth and Vigo (1997); W Wassan (2007); WS Wade and Salhi (2003); WN Wassan et al. (2008); G, E, X Nagy et al. (2013).

A Tabela 2 apresenta o GAP (\%) das diferentes proporções de clientes com coletas. Para o PRVB o algoritmo ficou com o custo médio $4,7 \%$ a mais em relação aos melhores resultados da literatura. Já no caso do PRVM o custo médio foi $6,2 \%$ maior. Para o valor de $\gamma=75 \%$ obteve-se redução de custo para todas as proporções de clientes. Para a proporção de clientes com coleta de $1 / 3$ e $1 / 2$ houve redução de custo para $\gamma=50 \%$. Na média final o aumento de custo foi de $2,7 \%$. O tempo de processamento foi inferior a 1 minuto em todas as instâncias e o mesmo não foi reportado na tabela de resultados.

Os resultados iniciais obtidos indicam que o procedimento de busca local precisa ser refinado, utilizando técnicas de path relinking ou a construção de uma heurística híbrida, utilizando busca Tabu ou GLS (Guided Local Search). 
Tabela 2 - Resultado Final \%GAP

\begin{tabular}{ccccc}
\hline $\begin{array}{c}\text { Proporção }(\mathrm{b} / \mathrm{n}): \\
\gamma\end{array}$ & $1 / 5$ & $1 / 3$ & $1 / 2$ & Total \\
\hline $100 \%$ & 5.2 & 4.7 & 4.2 & 4.7 \\
$75 \%$ & -1.1 & -4.7 & -3.3 & -3.0 \\
$50 \%$ & 4.4 & -0.1 & -0.2 & 1.4 \\
$25 \%$ & 3.8 & 3.7 & 0.0 & 2.5 \\
$10 \%$ & 5.8 & 4.5 & 6.8 & 5.7 \\
$0 \%$ & 5.6 & 4.6 & 8.4 & 6.2 \\
Total & 3.9 & 1.9 & 2.4 & 2.7 \\
\hline
\end{tabular}

\section{CONCLUSÕES E SUGESTÕES}

Nesse artigo foi apresentado o problema de roteirização de veículos com multicompartimentos com coleta e entrega mista restrita (PRVCEMR), com frota heterogênea. $\mathrm{O}$ mesmo é uma generalização do problema de roteirização de veículos com entregas e coletas, possibilitando o decisor a escolher o quanto de capacidade livre do veículo será aceito antes de começar a fazer as coletas. A restrição de multi-compartimentos foi incorporada ao problema, dando a possibilidade de resolver casos reais de roteirização, na qual a divisão do veículo por tipos de produtos é requisitada. Destaca-se que até o presente momento não existe nenhum trabalho tratando desse problema.

Foi desenvolvida uma heurística GRASP para a solução do PRVECMR, onde um procedimento de verificação de viabilidade foi implementado para tornar mais eficiente o tempo de verificação de inserção de um cliente na rota. A eficiência do algoritmo foi testada e o mesmo mostrou desempenho compatível com outros resultados da literatura. $\mathrm{O}$ procedimento de busca local precisa ser melhorado e a construção de uma heurística híbrida utilizando busca Tabu ou GLS (Guided Local Search) está sendo estudado, apesar da tendência de aumento do tempo de solução. A adaptação das instâncias da literatura para mais de um compartimento está em elaboração. Destaca-se que não existem instâncias disponíveis para o caso de coleta e entrega com veículos de mais de um compartimento.

Por fim, a integração com o roteirizador GeoRota web (http://www.loggeo.net/blog/ geo-rota/) está sendo implementada, dando a possibilidade de uso em empresas que necessitem otimizar suas rotas.

\section{REFERÊNCIAS BIBLIOGRÁFICAS}

1. BRANDÃO, J. A new tabu search algorithm for the vehicle routing problem with backhauls. European Journal of Operational Research, v. 173, p. 540-555, 2006.

2. CASCO, D. O.; GOLDEN, B. L.; WASIL, E. A. Vehicle routing with backhauls: models, algorithms and case studies. In A. A. Assad (Ed.). Vehicle routing: Methods and studies. Amsterdam: Elsevier, p. 127-147, 1988. 
3. CHAOVALITWONGSE, W.; KIM, D.; PARDALOS, P. M. GRASP with a new local search scheme for vehicle routing problems with time windows. Journal of Combinatorial Optimization, v. 7, n. 2, p. 179-207, 2003.

4. CRISPIM J.; BRANDÃO J.. Metaheuristics applied to mixed and simultaneous extensions of vehicle routing problems with backhauls. Journal of the Operational Research Society, v. 56(7), p. 1296-1302, 2005.

5. DEIF, I.; BODIN, L. Extension of the Clarke and Wright algorithm for solving the vehicle routing problem with backhauling. In A. E. Kidder (Ed.). Proceedings of the conference on software uses in transportation and logistics management, p. 75-96, 1984.

6. EL FALLAHI, A.; PRINS, C.; CALVO, R. W. A memetic algorithm and a tabu search for the multi-compartment vehicle routing problem. Computers \& Operations Research, v. 35, n. 5, p. 1725-1741, 2008.

7. FEO, T. A.; RESENDE, M.G.C. Greedy Randomized Adaptive Search Procedures. Journal of Global Optimization, v. 6, p. 109-133, 1995.

8. GAJPAL, Y.; ABAD, P. L. Multi-ant colony system (MACS) for a vehicle routing problem with backhauls. European Journal of Operational Research, v. 196, p.102$117,2009$.

9. GOETSCHALCKX, M.; JACOBS-BLECHA, C. (1989). The vehicle routing problem with backhauls. European Journal of Operational Research, v. 42, p. 39-51.

10. HALSE, K. Modeling and solving complex vehicle routing problems. Lyngby: Technical University of Denmark, 1992. PhD thesis, Institute of Mathematical Statistics and Operations Research.

11. KONTORAVDIS, G.; BARD, J. F. A GRASP for the vehicle routing problem with time windows. ORSA Journal on Computing, v. 7, n. 1, p. 10-23, 1995.

12. LAPORTE, G.; M. GENDRAU, J.Y; POTVIN, F. S. Classical and modern heuristics for the vehicle routing problem. International Transaction in Operational Research, v. 7 (4/5), p. 285-300, 2000.

13. MARTELLO S., TOTH P. Lower Bounds and Reduction Procedures for the Bin Packing Problem. Discrete Applied Mathematics, v. 28, p. 59-70, 1990.

14. MINGOZZI, A.; GIORGI, S.; BALDACCI, R. An exact method for the vehicle routing problem with backhauls. Transportation Science, v. 33, p. 315-329, 1999.

15. NAGY, G.; SALHI, S. Heuristic algorithms for single and multiple depot vehicle routing problems with pickups and deliveries. European Journal of Operational Research, v. 162, p. 126-141, 2005.

16. NAGY, G.; WASSAN, N. A.; SALHI, S. The vehicle routing problem with restricted mixing of deliveries and pickups. Journal of Scheduling, v. 16, n. 2, p. 199-213, 2013.

17. PARRAGH, S. N.; DOERNER, K. F.; HARTL, R. F. A survey on pickup and delivery problems. Part I: Transportation between customers and depot. Journal für Betriebswirtschaft, v. 58, p. 21-51, 2008. 
18. ROPKE, S.; PISINGER, D. A unified heuristic for a large class of vehicle routing problems with backhauls. European Journal of Operational Research, v. 171, p. 750$775,2006$.

19. SALHI S.; NAGY G. A cluster insertion heuristic for single and multiple depot vehicle routing problems with backhauling. Journal of the Operational Research Society, v. 50, p. 1034-1042, 1999.

20. TOTH, P.; VIGO, D. An exact algorithm for the vehicle routing problem with backhauls. Transportation Science, v. 31, p. 372-385, 1997.

21. TURKENSTEEN, M.; HASLE, G.. Combining pickups and deliveries in vehicle routing - An assessment of carbon emission effects. Transportations Research Part $C$, v. 80, p. 117-132, 2017.

22. TÜTÜNCÜ, G. Y.; CARRETO, C. A. C.; BAKER, B. M. A visual interactive approach to classical and mixed vehicle routing problems with backhauls. Omega, v. 37, p. 138$154,2009$.

23. WADE, A. C.; SALHI, S. An investigation into a new class of vehicle routing problem with backhauls. Omega, v. 30, p. 479-487, 2002.

24. WADE, A. C.; SALHI, S. An ant system algorithm for the mixed vehicle routing problem with backhauls. In M. G. Resende \& J. P. de Sousa (Eds.). Metaheuristics: Computer decision-making New York: Kluwer, 2003, p. 699- 719.

25. WASSAN, N. A. Reactive tabu adaptive memory programming search for the vehicle routing problem with backhauls. Journal of the Operational Research Society, v. 58, p. 1630-1641, 2007.

26. WASSAN, N. A.; NAGY, G.; AHMADI, S. A heuristic method for the vehicle routing problem with mixed deliveries and pickups. Journal of Scheduling, v. 11, p.149-161, 2008 . 